\title{
Low-order Continuous-time Robust Repetitive Control: Application in Nanopositioning
}

\author{
Arnfinn A. Eielsen,*, J. Tommy Gravdahla ${ }^{\mathrm{a}}$, Kam K. Leang ${ }^{\mathrm{b}}$ \\ ${ }^{a}$ Dept. of Engineering Cybernetics, Norwegian Univ. of Science and Technology, 7491 Trondheim, Norway \\ ${ }^{b}$ Dept. of Mechanical Engineering, Univ. of Utah, Salt Lake City, Utah, USA
}

\begin{abstract}
A low-order repetitive control (RC) design in continuous-time for nanopositioning applications is presented. It focuses on achieving high performance and sufficient robustness to uncertainties. The design is mainly applicable to analog implementation, but due to the exceptionally low order, it also results in a fast and efficient digital implementation. Experimental results for an analog implementation using a bucket brigade device (BBD), as well as a digital implementation, is presented. RC can provide fast and accurate tracking of periodic reference signals, which is useful in many scanning probe microscopy and nanofabrication applications.
\end{abstract}

Keywords: repetitive control; nanopositioning; scanning probe microscopy; piezoactuators; nanofabrication

\section{Introduction}

Nanopositioning stages often require control laws with the ability to track periodic reference signals with high accuracy, e.g. in scanning probe microscopy and nanofabrication. Such signals occur in applications such as raster scanning, pick-andplace operations, and mass-production of features [1, 2, 3].

Repetitive control (RC) is ideally suited for periodic signals. It is based on the internal model principle [4]: tracking or rejection of periodic exogenous signals can be achieved by embedding a periodic signal model in the control loop. A periodic signal model can be efficiently implemented using a time-delay inside of a positive feedback loop [5, 6, 7]. An important feature of $\mathrm{RC}$ is that as long the overall control loop is stable the $\mathrm{RC}$ scheme is invariant to changes in plant dynamics, subject to the accuracy of the signal model. To guarantee stability, the control law filters must ensure sufficient robustness. The requirement for robustness impacts on the accuracy of the signal model, as one of the most common methods to introduce robustness in $\mathrm{RC}$ is to limit the bandwidth of the signal model [6]. However, any linear control law without a periodic signal model, even a bandwidth-limited one, will not achieve the same level of performance for periodic signals.

Recently, RC has been introduced for nanopositioning systems $[8,9,10,11]$. For periodic references, due to the high degree of invariance to changes in plant dynamics and the ability to reject periodic disturbances, $\mathrm{RC}$ can address the challenges posed by state-of-the-art mechanically stiff nanopositioner-designs. Such systems often have lightly damped vibration modes, and use piezoelectric actuators which introduces hysteresis and creep [12]. Hysteresis and creep are the main sources of uncertainty, as these phenomena change the effective system gain de-

\footnotetext{
* Corresponding author A. A. Eielsen. Tel. +47 40244954.

Email addresses: eielsen@itk.ntnu.no (Arnfinn A. Eielsen), gravdahl@itk.ntnu.no (J. Tommy Gravdahl), kam.k.leang@utah.edu (Kam K. Leang)
}

pendent on input voltage offset, range, and frequency [2]. Hysteresis is also the main source of disturbance, as it generates harmonic distortion on the input for a given stationary excitation signal [13]. The disturbance is then periodic with the same fundamental frequency as the excitation signal, and RC can then provide good rejection. Additional uncertainty is introduced in applications, as it is typically required to move payloads of various masses, thus the vibration modes and the effective gain of the mechanical structure changes every time a new payload is attached. Other effects that introduce uncertainty are inherent variations in piezoelectric actuators, where the effective system gain changes due to temperature, depolarization, and aging.

Robustness for RC is usually taken to mean robustness towards uncertainties in the fundamental frequency of exogenous signals (robust performance), or robustness towards plant modeling uncertainty (robust stability). Methods to improve performance if there is uncertainty or variation in the fundamental frequency of exogenous signals have been proposed in $[14,15,16,9]$. Such methods are not applicable in this work, as the reference signal period is considered to be known and accurate and the main disturbance is due to hysteresis which generates harmonic distortion dependent on the reference signal [13].

Robustness towards plant uncertainty has been studied in [6, $7,17,18,19,20]$. The most common method to introduce robustness in RC is to limit the bandwidth of the signal model. This corresponds to the modified RC system in [6], where a lowpass filter is used to limit the bandwidth and hence relax the stability condition. A robust stability condition is found in [7], where an additive uncertainty weight is considered. Modelmatching techniques from robust control theory [21] is applied in $[17,18,19]$ to synthesize robust control law filters suitable for RC. Model-matching is also proposed in [6] for control law filters synthesis, but without considering robustness weights. In [20] robustness is introduced by accounting for uncertainty via a worst-case plant frequency response generated from measurements. An approximate discrete-time plant inverse filter is 
then found using system identification techniques. Control law filter synthesis using model-matching or system identification system identification techniques are not suited to the approach taken in this work, as there are constraints on filter order and structure.

RC is very similar to iterative learning control (ILC) [22, 23, 24, 25]. The main difference between ILC and RC is that ILC is a finite time problem where the initial values of the states are reset between each iteration. ILC can be applied to unstable systems and it does not require convergence in the solution of the input signal sequence. However, when applying RC it is not required to solve the initial value problem for each iteration step and it can be implemented using analog devices. For convenience, RC can also be plugged into an existing feedback loop to enhance performance with minimal changes to an existing control system [8].

\subsection{Contribution}

The aim of this work it to synthesize a low-order continuoustime robust $\mathrm{RC}$ scheme which can yield high performance and is suitable for analog implementation. This is achieved using a robust damping and tracking control law in combination with a plug-in type continuous-time repetitive control (RC) scheme. Specifically, a robust stability criterion and a tuning procedure for the RC scheme is proposed, and an inexpensive analog implementation of the scheme is presented. This work is an updated and expanded version of [26, 27].

\subsection{Outline}

The lightly damped vibration modes and the hysteresis effect present in many nanopositioning systems can degrade performance and make it difficult to obtain a stable RC system [28]. A modified integral control law [29] is therefore designed and used to mitigate the effects of vibration modes and hysteresis. This kind of control law can be described as a damping and tracking control law. Examples of such control laws applied to nanopositioning systems can be found in [30, 31, 32, 33, 34, 35]. Any of these control laws can provide an approximately flat frequency response for the complementary sensitivity function [29], which is one of the features used to reduce implementation complexity in the proposed control scheme. The particular control law used in this work is chosen because it provides the lowest order implementation and incorporates the anti-aliasing and reconstruction filters needed in the system to good effect.

The modified integral control law is combined with continuous-time RC for tracking of periodic references. Robust stability is considered via the selection of a multiplicative uncertainty weight. In order to reduce implementation complexity, the flat frequency response feature is exploited by approximating the complementary sensitivity function with an all-pole Butterworth filter. A Butterworth filter is by definition a filter which provides a maximally flat frequency response, or equivalently, uniform sensitivity in the passband [36]. An optimization problem is solved to find a DC-gain and a cut-off frequency for this Butterworth filter that ensures robust stability with the given multiplicative uncertainty weight. By using two all-pole filters, one for the approximation of the complementary sensitivity function and one to limit the bandwidth of the signal model, the inverse of the approximation combined with the low-pass filter produces a biproper transfer function. The biproper transfer function can then be implemented using a single filter with one input and two outputs, reducing implementation complexity.

Thus, the overall control scheme has exceptionally low order; simplifying the implementation process. A digital and an analog implementation is presented. The digital implementation uses standard digital signal processing (DSP) equipment, and the analog implementation is realized using regular analog filters and a bucket brigade device (BBD) [37, 38, 39], which provides the required time-delay. The use of BBDs for $\mathrm{RC}$ have previously been investigated [40,41], but using different control law structures and not for motion control. The digital implementation serves as a reference implementation for the subsequent analog implementation.

Experimental results are presented to demonstrate the effectiveness of the overall control scheme, where the proposed control system is applied to a custom-designed piezo-based nanopositioning system.

\subsection{Assessment of Experimental Performance}

In [29] several damping and tracking control laws have been surveyed for a similar system as the one considered here. Damping and tracking control laws typically only incorporate integral action, thus only providing asymptotic tracking for constant references. One of the best performing methods in [29], synthesized using model reference control (MRC), yielded a maximum error (ME) of $16 \%$ and a root-mean-square error (RMSE) of $11 \%$ for a $80-\mathrm{Hz}$ triangle wave reference signal with $1-\mu \mathrm{m}$ amplitude when there was a dominant vibration mode at $1660 \mathrm{~Hz}$. The corresponding figures for the modified integral control law used in this work applied to the system in [29] were an ME of $24 \%$ and an RMSE of $20 \%$. In this work, an ME of $12 \%$ and an RMSE of $12 \%$ was achieved using the modified integral control law for a $25-\mathrm{Hz}$ modified triangle wave reference signal with 13.5- $\mu \mathrm{m}$ amplitude when there is a dominant vibration mode at $704 \mathrm{~Hz}$.

When applying RC, the tracking performance is significantly improved. In this work, the analog implementation achieved an ME of $0.63 \%$ and an RMSE of $0.31 \%$ for a $50-\mathrm{Hz}$ triangle wave reference signal with $15-\mu \mathrm{m}$ amplitude when there is a dominant vibration mode at $704 \mathrm{~Hz}$. This constitutes an improvement of two orders of magnitude compared to the case when only applying a damping and tracking control law.

Experimental results for a similar system using discretetime RC can be found in [11]. Comparing the results, the presented continuous-time scheme will perform on par with the common discrete-time RC implementation that uses high-order zero-phase tracking error control (ZPETC) model-inversion and signal model bandwidth limitation using zero-phase filtering $[42,20]$. The discrete-time implementation in [11] achieved an ME of $0.83 \%$ and an RMSE of $0.10 \%$ for a $40-\mathrm{Hz}$ triangle wave reference signal with $5-\mu \mathrm{m}$ amplitude when there is a dominant vibration mode at $520 \mathrm{~Hz}$. 


\section{System Description and Modeling}

\subsection{Mechanical Model}

The nanopositioning stage used in this work is shown in Fig. 1, where the serial-kinematic motion mechanism is designed such that the first vibration mode is dominant and occurs in the actuation direction (piston mode). This is one of the most common design approaches. More details on the design of such stages can be found in [43, 12]. A free-body diagram for the mechanism is shown in the inset image in Fig. 1. It translates to the second-order differential equation (" $x$ " denotes $x$-direction)

$$
m_{x} \ddot{q}_{x}(t)+c_{x} \dot{q}_{x}(t)+k_{x} q_{x}(t)=f_{x}(t),
$$

where $m_{x}(\mathrm{~kg})$ is the mass of the sample platform and attached payload, $c_{x}\left(\mathrm{~N} \mathrm{~s} \mathrm{~m}^{-1}\right)$ is the damping coefficient, and $k_{x}\left(\mathrm{~N} \mathrm{~m}^{-1}\right)$ is the spring constant.

The piezoelectric actuator is modeled as a force transducer, generating a force proportional to the applied voltage [35]. The applied force from the piezoelectric actuator $f_{x}(\mathrm{~N})$ is

$$
f_{x}(t)=\beta u_{a}(t)+d_{u}(t)
$$

where $\beta\left(\mathrm{N} \mathrm{V}^{-1}\right)$ is the effective gain of the piezoelectric actuator from voltage to force, and $u_{a}(t)(\mathrm{V})$ is the applied voltage. The piezoelectric actuator will introduce hysteresis and creep when driven by a voltage signal. It is a reasonable assumption to consider this behavior as a bounded disturbance added to the input, represented by the term $d_{u}(t)$ [13].

Denoting the output $y=q_{x}$, the transfer-function for the nanopositioning stage from the voltage input $u_{a}$ is

$$
G(s)=\frac{y(s)}{u_{a}(s)}=\frac{b_{0}}{s^{2}+a_{1} s+a_{0}}=\frac{b_{0}}{s^{2}+2 \zeta \omega_{0} s+\omega_{0}^{2}},
$$

where $b_{0}=\beta / m\left(\mathrm{~m} \mathrm{~s}^{-2} \mathrm{~V}^{-1}\right), a_{0}=k / m\left(\mathrm{~s}^{-2}\right), a_{1}=c / m$ $\left(\mathrm{s}^{-1}\right), \zeta=c / 2 \sqrt{m k}$, and $\omega_{0}=\sqrt{k / m}\left(\mathrm{~s}^{-1}\right)$.

The frequency response for the $x$-axis is recorded using a SR780 Dynamic Signal Analyzer from Stanford Research Systems using 200-mV RMS bandwidth-limited white noise excitation. It is displayed in Fig. 2. The model (3) is fitted to the

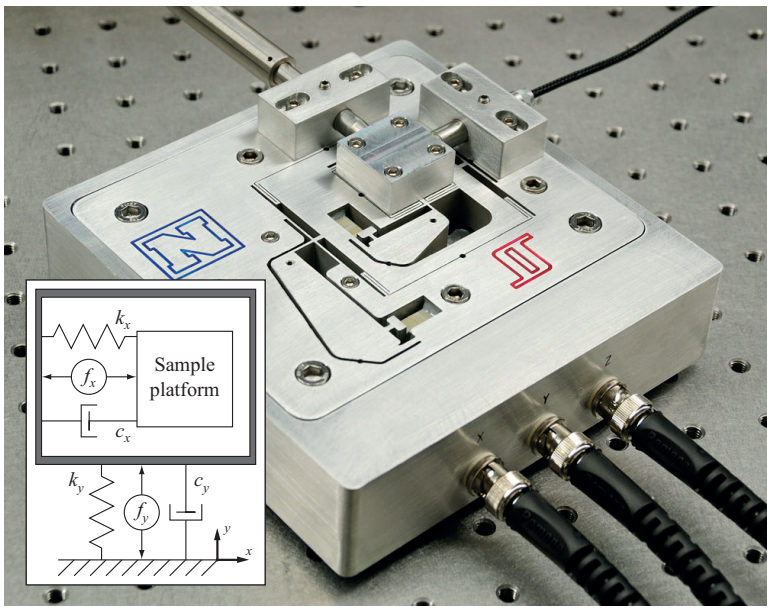

Figure 1: Custom flexure-guided nanopositioning stage.

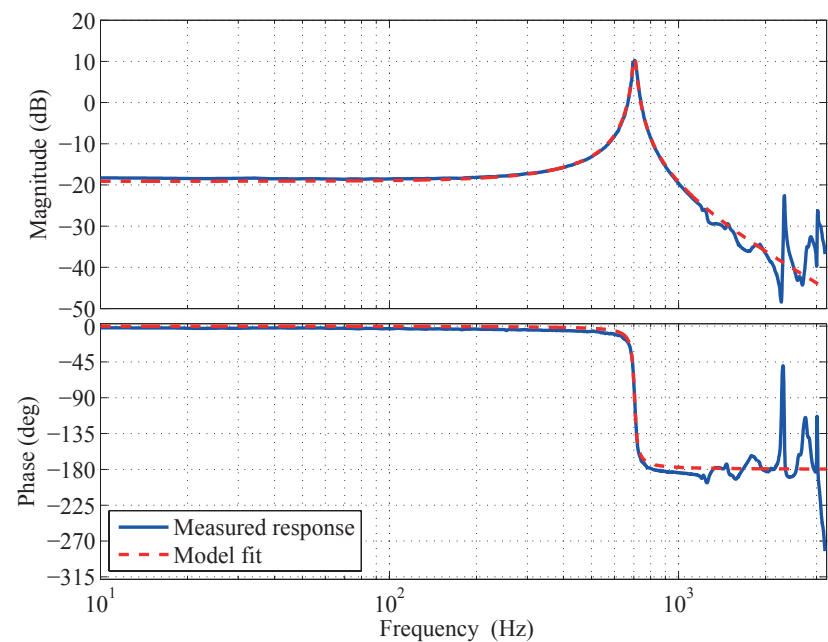

Figure 2: Frequency response: measured data and fitted model (3).

frequency response data using the MATLAB System Identification Toolbox, and the resulting parameter values are presented in Tab. 1. The response of the model (3) using these parameters is also displayed in Fig. 2 for comparison, where good agreement is achieved up to approximately $2 \mathrm{kHz}$.

Table 1: Identified parameters for the model (3).

\begin{tabular}{ccc}
\hline \hline Parameter & Value & Unit \\
\hline$b_{0}$ & $2.17 \cdot 10^{6}$ & $\mu \mathrm{m} \mathrm{s}^{-2} \mathrm{~V}^{-1}$ \\
$a_{0}$ & $1.96 \cdot 10^{7}$ & $\mathrm{~s}^{-2}$ \\
$a_{1}$ & 144 & $\mathrm{~s}^{-1}$ \\
$\zeta$ & $16.3 \cdot 10^{-3}$ & 1 \\
$\omega_{0}$ & $2 \pi \cdot 704$ & $\mathrm{~s}^{-1}$ \\
\hline \hline
\end{tabular}

\subsection{Uncertainty}

As can be seen from Fig. 2, the response of the first vibration mode is well approximated by the model (3), and it is sufficient to describe the dominant dynamics of the system. In order to assess the robustness properties of the control scheme, the uncertainties of the system must be accounted for.

In Fig. 2, vibration modes above the dominant (first) vibration mode can be seen. Higher-order vibration modes are likely to have shapes and directions that can make them difficult to control using the mounted actuator (along the $x$-direction). The control law therefore needs sufficient attenuation at higher frequencies to avoid excitation of the higher order vibration modes.

Eq. (3) also has uncertainty with regards to the parameters. Most susceptible to change is the effective gain $\beta$, and $\beta$ is mainly determined by the piezoelectric material used in the actuator, amount of polarization, and driving voltage amplitude; as the amount of displacement generated changes with voltage amplitude due to hysteresis. This is demonstrated in Fig. 3, where the relative change of the low-frequency gain $b_{0} / a_{0}$ is recorded as a function of input voltage amplitude. The relative change in gain is found to be up to $80 \% \approx-2 \mathrm{~dB}$ at an amplitude of $100 \mathrm{~V}$ compared to the gain at $50 \mathrm{mV}$. 


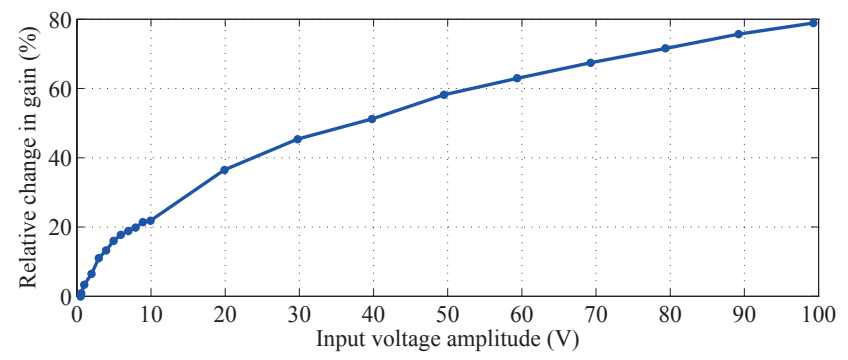

Figure 3: Relative change due to amplitude for a $10-\mathrm{Hz}$ sinusoidal voltage signal.

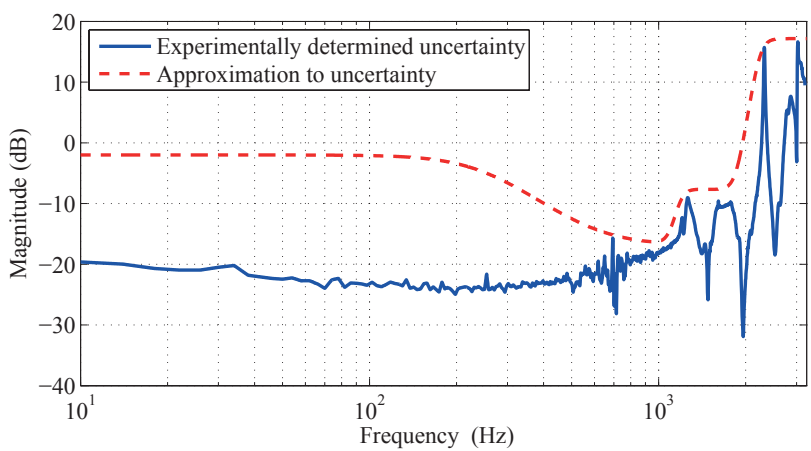

Figure 4: Open-loop uncertainty weight $w_{G}(s)$.

The peak amplitude response of the first and higher order vibration modes did, however, not seem to change as a function of input voltage amplitude. It is pointed out that this is difficult to measure over a large domain of voltage amplitudes, as fairly low input amplitudes lead to excessively large displacements when the system is excited using frequencies close to the first resonance frequency. A reasonable assumption in this case would then be that the uncertainty in gain diminishes when approaching the first resonance from below.

To assess the robustness of the proposed control scheme, the uncertainty of the system model is taken into account as a multiplicative perturbation to the nanopositioner dynamics,

$$
G_{p}(s)=G(s)\left[1+w_{G}(s) \Delta_{G}(s)\right] ;\left|\Delta_{G}(j \omega)\right| \leq 1 \forall \omega .
$$

The uncertainty weight $w_{G}(s)$ is determined experimentally. An over-bounding transfer-function is proposed, incorporating the gain uncertainty in Fig. 3 and the measured uncertainty due to the missing vibration modes in (3). The measured uncertainty and proposed overbounding uncertainty weight are shown in Fig. 4.

\section{Control Structure}

The control scheme combines plug-in RC and a damping and tracking control law, consisting of an integral (I) or a proportional-integral (PI) control law, and the anti-aliasing and reconstruction filters. This is shown in Fig. 5, which displays the structure used for the digital and the analog implementation.

\subsection{Repetitive Control (RC)}

$\mathrm{RC}$ will track or reject arbitrary periodic signals of a fixed period $\tau_{p}$, by embedding a model of the reference signal $r$ or

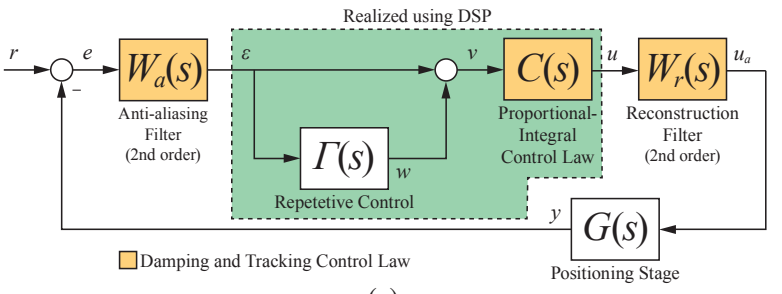

(a)

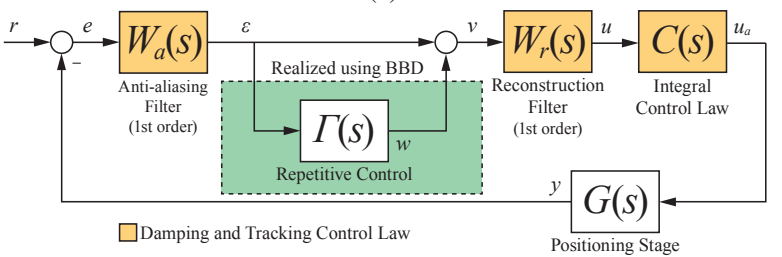

(b)

Figure 5: System diagrams: (a) DSP realization. (b) BBD realization.

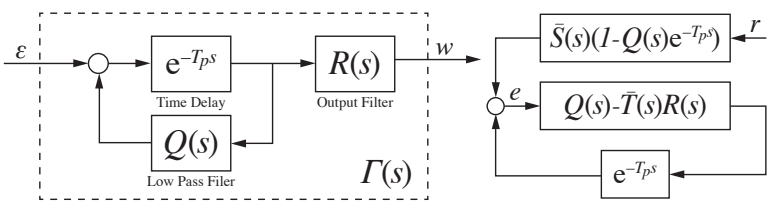

(a)

(b)

Figure 6: (a) Plug-in RC scheme. (b) Equivalent representation of (9).

disturbance signal $d$ in the control law. Such a model can be generated in a computationally efficient manner using a timedelay inside of a positive feedback loop [6, 7]. The transferfunction for the RC configuration shown in Fig. 6a is given as

$$
\frac{w}{\varepsilon}(s)=\Gamma(s)=\frac{R(s) \mathrm{e}^{-\tau_{p} s}}{1-Q(s) \mathrm{e}^{-\tau_{p} s}},
$$

where $Q(s)$ is a unity DC-gain low-pass filter, and $R(s)$ is an output filter, defined below. This is variation of the modified RC system in [6]. The filter $Q(s)$ is used to limit the bandwidth of the signal model. The low-pass filter $Q(s)$ shifts all the poles into the complex left half-plane with an amount dependent on frequency, degrading the nulling property of RC at the fundamental and harmonic frequencies of the reference. Steps to improve on this situation are discussed in Section 4.2.2. By implementing $Q(s)$ as a linear-phase FIR filter, this problem can be avoided [42, 20,11], but is not an option for an analog circuit.

By inspection of Fig. 5, the closed-loop sensitivity function for the overall system is found as

$$
\frac{e}{r}(s)=S(s)=\frac{1}{1+\bar{G}(s) C(s)+\bar{G}(s) C(s) \Gamma(s)}=\frac{1}{\Delta(s)},
$$

where $\bar{G}(s)=W_{r}(s) G(s) W_{a}(s)$. The stability of the closedloop system is determined by the denominator

$$
\Delta(s)=1+\bar{G}(s) C(s)+\bar{G}(s) C(s) \Gamma(s) .
$$

Consider the sensitivity $\bar{S}(s)$ and complementary $\bar{T}(s)$ sensitivity function excluding the RC scheme

$$
\bar{S}(s)=\frac{1}{1+C(s) \bar{G}(s)} \text { and } \bar{T}(s)=C(s) \bar{G}(s) \bar{S}(s) .
$$


By inserting the expression for $\Gamma(s)$, multiplying the numerator and denominator of $1 / \Delta(s)$ by $\bar{S}(s)$, and rearranging, the sensitivity function for the closed-loop system with RC becomes

$$
S(s)=\frac{1}{\Delta(s)}=\frac{\bar{S}(s)\left(1-Q(s) \mathrm{e}^{-\tau_{p} s}\right)}{1-(Q(s)-\bar{T}(s) R(s)) \mathrm{e}^{-\tau_{p} s}} .
$$

With reference to Fig. 6b, it can be seen that given a bounded reference $r(t)$ and stable transfer-functions $\bar{S}(s)$ and $Q(s)$, the small-gain theorem provides the criterion for the stability of the closed-loop system as [6]

$$
\|Q(s)-\bar{T}(s) R(s)\|_{\infty}<1=0 \mathrm{~dB},
$$

where it is noted that $\left|\mathrm{e}^{-j \tau_{p} \omega}\right|=1 \forall \omega \in \mathbb{R}$.

The output filter $R(s)$ is constructed as

$$
R(s)=W_{T}^{-1}(s) Q(s),
$$

introducing a stable all-pole filter $W_{T}(s)$. Using a unity DCgain, all-pole, low-pass filter $Q(s)$, the criterion

$$
\left\|Q(s)\left(1-W_{T}^{-1}(s) \bar{T}(s)\right)\right\|_{\infty}<1=0 \mathrm{~dB}
$$

can be used for selecting an appropriate filter $W_{T}(s)$. It will be chosen to match the response of $\bar{T}(s)$ sufficiently to satisfy the stability criterion, i.e. $W_{T}(s) \approx \bar{T}(s)$. The selection of $W_{T}(s)$ is described in Sec. 4.2.1. By this particular choice, $W_{T}(s)$ and $Q(s)$ being all-pole filters, $R(s)$ and $Q(s)$ can be realized using a single filter with one input and two outputs, as shown in Sec. 5.3.4.

To assess the robustness of the $\mathrm{RC}$, a multiplicative perturbation for the closed-loop complementary sensitivity is used:

$$
\bar{T}_{p}(s)=\bar{T}(s)\left(1+w_{T}(s) \Delta_{T}(s)\right) ; 0<\left|\Delta_{T}(j \omega)\right| \leq 1 \forall \omega
$$

Incorporating the uncertainty weight $w_{T}(s)$ into the criterion (10) and applying the triangle inequality, a robust stability criterion is obtained:

$$
|Q(j \omega)-\bar{T}(j \omega) R(j \omega)|<1-\left|\bar{T}(j \omega) w_{T}(j \omega) R(j \omega)\right| .
$$

An estimate of the uncertainty weight $w_{T}(s)$ can be found using the uncertainty weight $w_{G}(s)$. Assuming that the uncertainty is due only to the plant, then, using $\bar{S}(s)+\bar{T}(s)=1$,

$$
w_{T}(s) \Delta_{T}(s)=\frac{\bar{S}(s) w_{G}(s) \Delta_{G}(s)}{1+\bar{T}(s) w_{G}(s) \Delta_{G}(s)},
$$

and by using (21) and the reverse triangle inequality, the closedloop uncertainty weight $w_{T}(s)$ is bounded from above by

$$
\left|w_{T}(j \omega)\right| \leq \frac{\left|\bar{S}(j \omega) w_{G}(j \omega)\right|}{1-\left|\bar{T}(j \omega) w_{G}(j \omega)\right|} .
$$

The bound in (15) can be conservative. In this work,

$$
\left|w_{T}(j \omega)\right| \approx\left|\bar{S}(j \omega) w_{G}(j \omega)\right|
$$

was empirically found to be a good approximation.

\subsection{Damping and Tracking Control Law}

The nanopositioning stage is a lightly damped structure; ref. Fig. 2. Inspecting the stability criterion (10) one can expect that peaks in the magnitude response of $\bar{T}(s)$ can reduce the applicable bandwidth for RC, depending on how well the output filter $R(s)$ is able to match the inverse closed-loop dynamics. Introducing a robust damping and tracking control law increases the robustness and bandwidth for the overall control scheme.

A simple, effective, and robust damping and tracking control law for a lightly damped structure can be obtained by modifying and optimally tuning an integral (I) or a proportional-integral (PI) control law [29]. Since both the BBD and DSP implementation utilizes sampling, and reconstruction and anti-aliasing filters must be present in order to mitigate aliasing effects. As the external clock signal driving the BBD bleed into the output signal, the reconstruction filter also serves to attenuate the clock signal noise. For the DSP implementation the reconstruction filter reduces quantization noise. By including these filters, an extra degree of freedom is added for the tuning of the control law, i.e., the cut-off frequency of the filters. As it turns out [29], the low-pass filters can approximate the effect that other damping and tracking control laws [35, 31, 30, 32, 33] introduces. Thus, by also adjusting the cut-off frequency $\omega_{c}$, damping can be introduced and the overall bandwidth increased. Since reconstruction and anti-aliasing filters must be present, this particular control law structure provides a minimal physical realization, which also uses the filters to good effect. The integral action also serves to suppress the hysteresis and creep nonlinearities.

The digital implementation uses a PI control law and two second-order low-pass Butterworth filters, whereas the analog implementation uses an I control law and two first-order lowpass filters, as discussed in Sec. 4.3.

The I and PI control laws are given as,

$$
C(s)=\frac{k_{i}}{s} \text { and } C(s)=\frac{k_{p} s+k_{i}}{s}
$$

where $k_{p}$ and $k_{i}$ are the proportional and integral gains. The reconstruction and anti-aliasing filters are here taken to be secondorder low-pass Butterworth filters

$$
W_{a}(s)=W_{r}(s)=\frac{\omega_{c}^{2}}{s^{2}+\sqrt{2} \omega_{c} s+\omega_{c}^{2}},
$$

for the digital implementation, and first-order low-pass filters

$$
W_{a}(s)=W_{r}(s)=\frac{\omega_{c}}{s+\omega_{c}}
$$

for the analog implementation. For these filters, $\omega_{c}$ is the cutoff frequency. For convenience they are chosen to be identical when tuning the control law. The modified I or PI control law are in either case denoted

$$
\bar{C}(s)=W_{a}(s) C(s) W_{r}(s) .
$$

The procedure for obtaining the optimal tuning of the control law applied to a flexible structure is described in Sec. 4.1. 


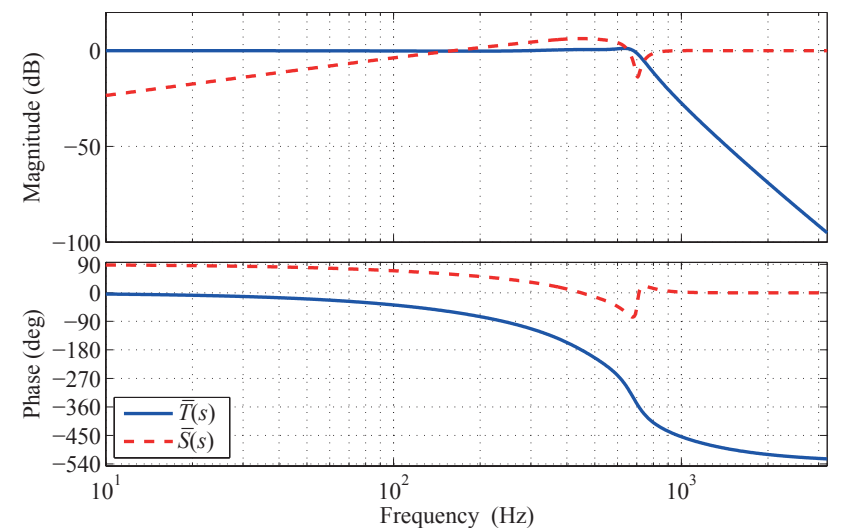

Figure 7: Frequency response for $\bar{T}(s)$ and $\bar{S}(s)$, for the PI control law and two second-order filters (digital implementation).

\section{Control Law Tuning and Analysis}

\subsection{Damping and Tracking Control Law}

As discussed in [29], a practical method to find optimal values for the cut-off frequency and the integral gain is to minimize

$$
J_{C}\left(k_{i}, \omega_{c}\right)=\left\|1-\left|\bar{T}\left(k_{i}, \omega_{c} ; j \omega\right)\right|\right\|_{2},
$$

where $\|\cdot\|_{2}$ denotes the frequency domain $L^{2}$-norm, truncated as needed. The minimization of (20) attempts to produce the flattest possible response for the complementary sensitivity function, i.e., an approximate Butterworth filter response. As larger values of $k_{p}$ resulted in poorer closed-loop response, this gain value was set to a fixed low value of $k_{p}=1 \mathrm{~V} / \mu \mathrm{m}$, in the case where the PI control law was used. Evaluating the costfunction (20)

$$
\begin{aligned}
& {\left[k_{i}{ }^{\star}, \omega_{c}{ }^{\star}\right]=\underset{k_{i}, \omega_{c}}{\arg \min }\left[J_{C}\left(k_{i}, \omega_{c}\right)\right]} \\
& \text { s.t. } \operatorname{Re}\left\{\lambda_{i}\right\} \in \mathbb{R}^{-} \wedge k_{i} \in \mathbb{R}^{+} \wedge \omega_{c} \in \mathbb{R}^{+} /\{0\},
\end{aligned}
$$

where $\lambda_{i}$ are the eigenvalues of the closed-loop system, resulted in the parameters presented in Tab. 2. The optimization problem can be solved in MATLAB using the Optimization Toolbox, e.g. with fminsearch, fminunc, fmincon, or by an exhaustive search. The latter is illustrated by the plot of the surface of the cost-function in the case when using the PI control law in a neighborhood of the optimal values; shown in Fig. 9.

The resulting frequency responses for the complementary sensitivity $\bar{T}(s)=\frac{y}{r}(s)$ and sensitivity function $\bar{S}(s)=\frac{e}{r}(s)$ for the two configurations of I or PI control law and first-order or second-order reconstruction and anti-aliasing filters are displayed in Figs. 7 and 8.

Robust stability with regards to the uncertainties displayed in Fig. 4 is determined by evaluating the criterion [21]

$$
\left\|w_{G}(s) \bar{T}(s)\right\|_{\infty}<1=0 \mathrm{~dB},
$$

and the results for the case of the PI control law (digital) are shown in Fig. 10. The control law allows for large uncertainty in high-frequency dynamics. This means that the presence of higher order modes will not interfere with the stability. The results are very similar for the I control law (analog).

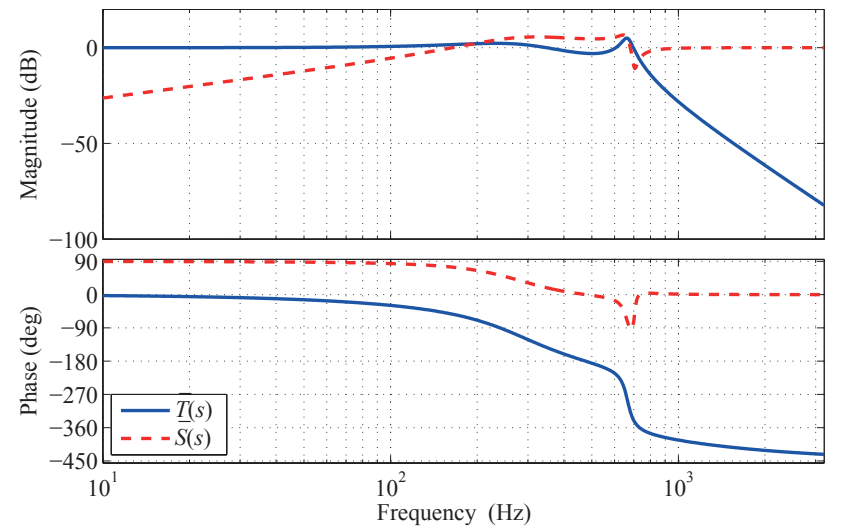

Figure 8: Frequency response for $\bar{T}(s)$ and $\bar{S}(s)$, for the I control law and two first-order filters (analog implementation).

Table 2: Optimal control law parameters.

\begin{tabular}{ccc}
\hline \hline & $k_{i}{ }^{\star}$ & $\omega_{c}{ }^{\star}$ \\
\hline PI control law (digital impl.) & $8380 \mathrm{~V} / \mathrm{s} \mu \mathrm{m}$ & $2 \pi \cdot 744 \mathrm{rad} / \mathrm{s}$ \\
I control law (analog impl.) & $11700 \mathrm{~V} / \mathrm{s} \mu \mathrm{m}$ & $2 \pi \cdot 495 \mathrm{rad} / \mathrm{s}$ \\
\hline \hline
\end{tabular}

\subsection{Repetitive Control}

\subsubsection{Choosing $R(s)$ and $Q(s)$}

Considering (12), $W_{T}(s)=\bar{T}(s)$ is the ideal choice, as this will produce the minimum of (12). Since RC performance is inherently robust against uncertainty due to the nulling property, a sufficient choice for $W_{T}(s)$ is a filter that attenuates $\bar{T}(s)$ enough to satisfy (12).

The complementary sensitivity function $\bar{T}(s)$ has relative degree $n_{r}=6$ for the digital implementation and $n_{r}=5$ for the analog implementation. As the frequency response for the complementary sensitivity function $\bar{T}(s)$ resembles that of a Butterworth filter for the two implementations, $W_{T}(s)$ is chosen to be a Butterworth filter of order $n_{r}$ with DC-gain $1 / k_{T}$, and it is then assumed that $W_{T}(s) \approx \bar{T}(s)$. An optimal choice for the cut-off frequency $\omega_{T}$ and DC-gain $k_{T}$ for $W_{T}(s)$ is found

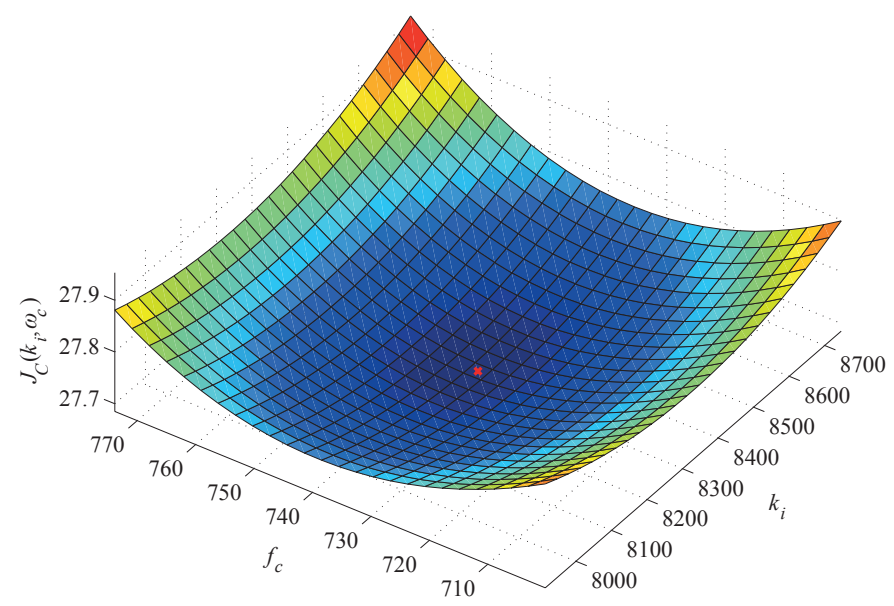

Figure 9: Optimal PI tuning. 


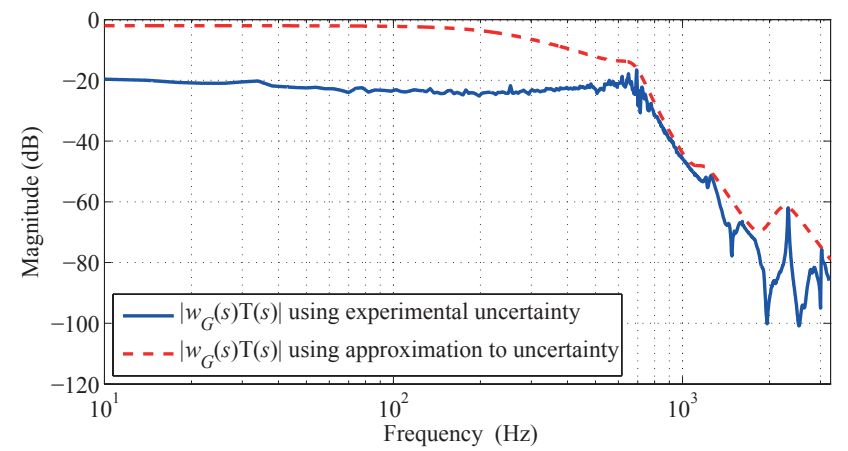

Figure 10: Evaluation of robust stability criterion $\left|w_{G}(j \omega) \bar{T}(j \omega)\right|$ for the modified PI control law.

minimizing the cost-function

$$
\begin{array}{r}
J_{T}\left(k_{T}, \omega_{T}\right)=\sup \left\{\mid Q(j \omega)-\bar{T}(j \omega) R\left(\omega_{T}, k_{T} ; j \omega\right)\right) \mid \\
\left.\left.+\mid \bar{T}(j \omega) w_{T}(j \omega) R\left(\omega_{T}, k_{T} ; j \omega\right)\right) \mid: \omega \in \mathbb{R}^{+}\right\},
\end{array}
$$

where $R\left(\omega_{T}, k_{T} ; j \omega\right)=W_{T}\left(\omega_{T}, k_{T} ; j \omega\right)^{-1} Q(j \omega)$. The costfunction must satisfy $J_{T}\left(k_{T}^{\star}, \omega_{T}^{\star}\right)<1$ in order for the system to be robustly stable. The closed-loop uncertainty weight $w_{T}(s)$, using experimental data and the overbounding approximation, is shown in Fig. 12 for the PI control law (digital). The overbounding approximation is used for the optimization. The results are similar for the I control law (analog).

The filter $Q(s)$ must be chosen before performing the optimization. It is chosen to be a unity DC-gain, all pole, Butterworth filter of order $n_{r}$, making $R(s)=W_{T}{ }^{-1} Q(s)$ a biproper filter. By this particular choice, $W_{T}(s)$ and $Q(s)$ being all-pole filters, $R(s)$ and $Q(s)$ can be realized using a single filter with two outputs, as shown in Sec. 5.3.4.

By inspection of Fig. 2, a cut-off frequency for $Q(s)$ is chosen in order to attenuate the second and higher order vibration modes, in order to satisfy (14). A cut-off frequency $\omega_{Q}=2 \pi \cdot 2000 \mathrm{rad} / \mathrm{s}$ provided sufficient attenuation. Minimizing (22) resulted in the parameters presented in Tab. 3. The surface of the cost-function for the PI control law case in a neighborhood of the optimal values is shown in Fig. 11.

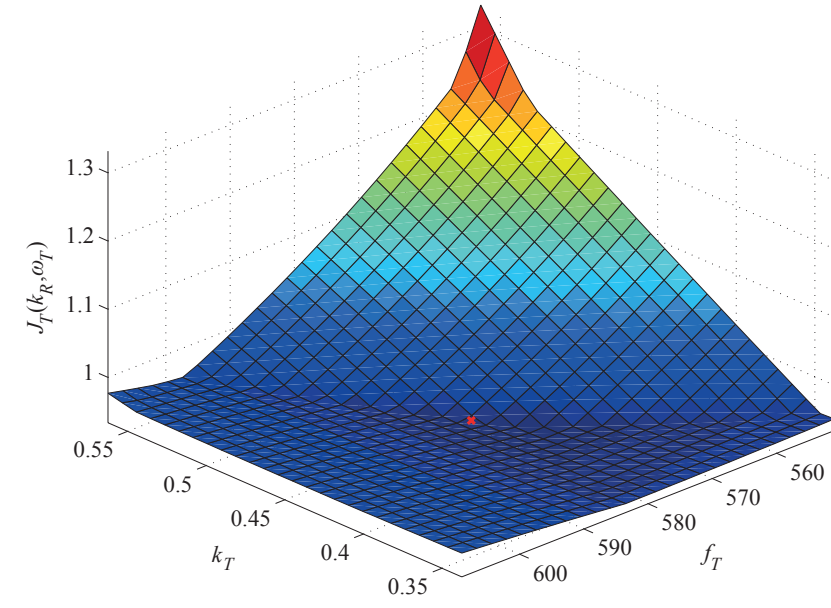

Figure 11: Optimal RC tuning.

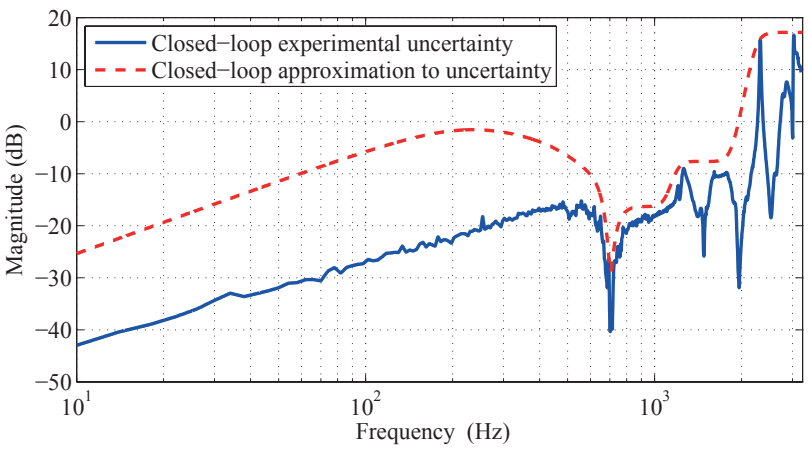

Figure 12: Closed-loop uncertainty weight $w_{T}(s)$ for the PI control law, using experimental data and as an overbounding transfer-function.

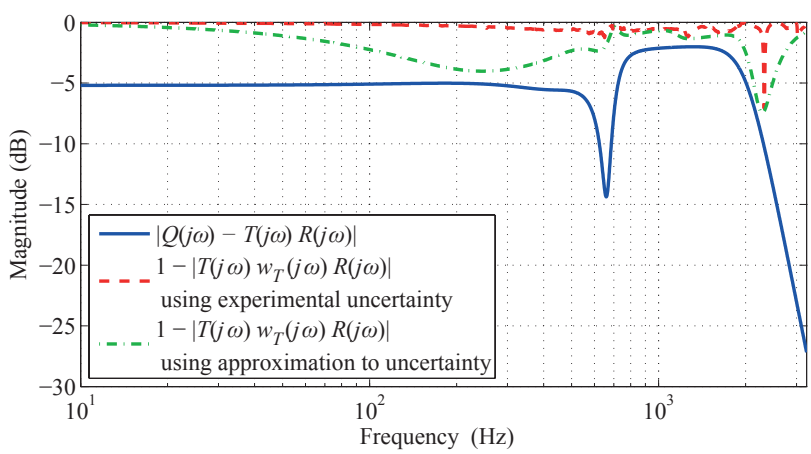

Figure 13: Evaluation of stability criteria (10) and (14) for the PI control law.

The evaluation of (10) and (14) is shown in Fig. 13 for the case of the PI control law, and demonstrates that the choice of $\omega_{Q}$ and the optimal values for $k_{T}$ and $\omega_{T}$ provide robust stability of the closed-loop system for the chosen uncertainty weight. The results are similar for case of the I control law.

Table 3: Optimal parameters for $W_{T}(s)$.

\begin{tabular}{ccc}
\hline \hline & $k_{T}{ }^{\star}$ & $\omega_{Q}{ }^{\star}$ \\
\hline PI control law (digital impl.) & $0.41 \mathrm{~V} / \mathrm{s} \mu \mathrm{m}$ & $2 \pi \cdot 580 \mathrm{rad} / \mathrm{s}$ \\
I control law (analog impl.) & $0.24 \mathrm{~V} / \mathrm{s} \mu \mathrm{m}$ & $2 \pi \cdot 530 \mathrm{rad} / \mathrm{s}$ \\
\hline \hline
\end{tabular}

\subsubsection{Tuning of $\tilde{\tau}_{p}$}

Inspecting the sensitivity function (9), note that the numerator contains the expression

$$
\tilde{Z}(s)=1-Q(s) \mathrm{e}^{-\tilde{\tau}_{p} s} .
$$

Using the expression $\tilde{r}(t)=Q(t) * r(t)$ to denote the convolution of the signal $r(t)$ with the impulse response $Q(t)$, this evaluates to

$$
\mathcal{L}^{-1}\left[\left(1-Q(s) \mathrm{e}^{-\tilde{\tau}_{p} s}\right) r(s)\right]=r(t)-\tilde{r}\left(t-\tilde{\tau}_{p}\right)
$$

when $r(t)=0 \forall t<0$ and $r(t)=r\left(t+\tilde{\tau}_{p}\right)$. Thus, disregarding the initial value response of the system, the reference is perfectly nulled in the error after one period $\tau_{p}$ of the reference and perfect tracking is obtained only if $Q(s)=1$. For the actual implementation $\tilde{r}(t)=Q(t) * r(t) \neq r(t)$. Since the filter $Q(s)$ alters the reference, perfect tracking can not be obtained. 
The residual error depends both on $Q(s)$ and the time-delay $\tilde{\tau}_{p}$. Tracking performance can be improved by adjusting $\tilde{\tau}_{p}$.

By filtering the intended reference by $\tilde{Z}(s)$, it is possible to numerically evaluate the amount of suppression, or nulling, of the reference. By adjusting $\tilde{\tau}_{p}=\tau_{p}-\delta$ by varying $\delta$, an optimal $\delta$ that produces the best tracking performance can be found minimizing

$$
J(\delta)=\left\|\mathcal{L}^{-1}(\tilde{Z}(s, \delta)) * r(t)\right\|_{2}, t \in\left[\tau_{p} m, \tau_{p}(m+1)\right]
$$

where $\|\cdot\|_{2}$ denotes the time domain $L^{2}$-norm, $\delta \in\left(0, \tau_{p}\right)$, and $m$ is a positive integer to make sure $\tau_{p} m$ is large enough for the transient response of $Q(s)$ to have died out. An efficient implementation of this cost function can be made by using Parseval's theorem after computing the stationary Fourier coefficients of a given reference signal filtered by $\tilde{Z}(s)$. Results are presented in Tab. 4. Note that on a digital platform, it is possible to implement time-delays with a non-integer multiple of the sampling time using Thiran-approximation [44]. For the analog implementation, since the exact value of $\tilde{\tau}_{p}$ is dependent on the tolerances of components used, the simplest solution is to adjust the fundamental frequency of the reference in order to minimize the stationary root-mean-square error (RMSE).

Table 4: Optimal $\delta$ given $f_{p}$ using 6th-order $Q(s)$ with $\omega_{Q}=2 \pi \cdot 2000 \mathrm{rad} / \mathrm{s}$.

\begin{tabular}{cc}
\hline \hline$f_{p}$ & $\delta^{\star}$ \\
\hline $25 \mathrm{~Hz}$ & $3.07709 \cdot 10^{-4} \mathrm{~s}$ \\
$50 \mathrm{~Hz}$ & $3.08076 \cdot 10^{-4} \mathrm{~s}$ \\
$100 \mathrm{~Hz}$ & $3.08384 \cdot 10^{-4} \mathrm{~s}$ \\
$200 \mathrm{~Hz}$ & $3.08982 \cdot 10^{-4} \mathrm{~s}$ \\
$400 \mathrm{~Hz}$ & $3.09129 \cdot 10^{-4} \mathrm{~s}$ \\
\hline
\end{tabular}

\subsection{Some Remarks on Control Law Order and Implementation}

Several practical factors were taken into account when choosing between a PI and I control law, and the order of the antialiasing and reconstruction filters. For the digital implementation, two second-order programmable filters were available for the experimental set-up, dictating the minimum order for these filters. Implementing arbitrary linear filters does not cost anything in terms of component count on a DSP system, thus a PI control law could be used. This meant that $\bar{T}(s)$ would have a relative degree of $n_{r}=6$, rather than $n_{r}=7$; reducing the order of the required $R(s)$ and $Q(s)$ filters. For the analog implementation, choosing an I control law meant that the component count would be minimal as it would remove the need for a summation stage. By choosing a PI control law the $R(s)$ and $Q(s)$ filters could have been fourth-order filters, but an operational amplifier section was available for the filter circuit that otherwise would not have been used; making it possible to implement a fifth-order filter at an overall lower component count.

With the chosen control structure, the minimal relative degree for $\bar{T}(s)$ is $n_{r}=4$. The combination of two first firstorder low-pass filters and a PI control law will produce this. As the anti-aliasing and reconstruction filters must be present as a part of the control system, any other control law synthesis should incorporate these into the plant dynamics in order to reduce model uncertainty and to increase performance. A standard model matching control law, such as model reference control (MRC), would in comparison produce a relative degree of $n_{r}=5$ for $\bar{T}(s)$, if integral action is included. Here, however, two fifth-order filters, as well as an integrator for the integral action, would be needed to implement the control law, thus increasing the number of needed integrators from one to eleven. Similarly, $\mathcal{H}_{\infty}$ or $\mathcal{H}_{2}$ control law synthesis, will produce a control law filter of minimum fourth-order, but an implementable control law filter would be of higher order, depending on the weights used in the synthesis. Similarly, any of the damping and tracking schemes, other than the one chosen in this work, as surveyed in [29], will increase the order of the control law $C(s)$. Thus, the order of the $R(s)$ and $Q(s)$ filters might not increase much, but the control law used to produce an approximate Butterworth (maximally flat) response for $\bar{T}(s)$ will be of significantly higher order and/or require a higher component count. It should also be noted that the $R(s)$ and $Q(s)$ filters in the implementation are constructed in such a way that they can be implemented as a single filter with one input and two outputs, further reducing implementation complexity and component count.

\section{Experimental Results and Discussion}

The performance and robustness of the control scheme and the digital and the analog implementations was validated by experiments. As it was simpler to adjust time-delay and scaling for maximum dynamic range when using the digital implementation, this was used for most of the results. The dynamic range in the case of the digital implementation was maximized by scaling input and output signals to take full advantage of the resolution of the analog-to-digital converter (ADC) and digital-toanalog converter (DAC). In the analog case, the dynamic range was maximized by adjusting the scaling of the signals going in and out of the bucket brigade device (BBD).

\subsection{Description of the Experimental System}

The system to which the both the digital and the analog implementation was applied consisted of an ADE 6810 gauge and ADE 6501 capacitive probe from ADE Technologies for measuring displacement, a Piezodrive PDL200 voltage amplifier, and the custom-made nanopositioner (see Fig. 1). The capacitive displacement measurement has a sensitivity of $k_{s}=1 / 5$ $\mathrm{V} / \mu \mathrm{m}$ and the voltage amplifier has a gain of $20 \mathrm{~V} / \mathrm{V}$.

\subsection{Digital Implementation}

Digital implementation used a dSPACE DS1103 hardwarein-the-loop system, programmed using MATLAB and Simulink via the Simulink Coder. Two Stanford Research Systems (SRS) SIM965 programmable filters were used as anti-aliasing and reconstruction filters, and two SRS SIM983 scaling amplifiers were used to scale the input and output signals from the DS1103 system. For numerical integration, the fourth-order Runge- 
Kutta scheme was used, and a sampling time of $T_{s}=10^{-5}$ $\left(f_{s}=100 \mathrm{kHz}\right)$ was used for all the experiments.

A modified triangle-wave was used as the reference: of the initial amplitude $\alpha$ of a regular triangle-wave, the fraction $h=\lambda / \alpha$ was left linear, and the signal near the extrema was replaced by a spline. This was done to reduce the spectral content to avoid saturations at high frequencies and amplitudes. In the implementation, a second-order spline and $h=90 \%$ was used.

\subsection{Analog Implementation}

\subsubsection{Rationale}

For a digital implementation, to obtain high accuracy and low noise, the ADCs and DACs used must feature high resolution and high sampling frequency. High resolution is required for a low bound on the achievable accuracy and to minimize quantization noise. The sampling frequency must be sufficiently high with respect to the bandwidth of dominant dynamics of the plant, and to minimize the power spectral density of the quantization noise. Thus, the DSP platform used must handle high data rates and support floating-point arithmetic or large word size fixed-point arithmetic. Programming the required filtering algorithms can also be time-consuming, and for some platforms (notably field-programmable gate arrays) proprietary compilers are required. The implementation can be simplified by using ready-made hardware-in-the-loop systems, as has been done in this work, albeit at a higher overall cost for the system.

The analog implementation uses analog filters and a BBD for the realization of RC. Low-noise BBDs can match 16-bit ADCs and DACs in terms of noise performance. In terms of required equipment, this is an inexpensive and simpler alternative to DSP. The main noise source when using a BBD is the external clock signal. A reconstruction filter with sufficient attenuation at and above the driving clock signal frequency is required. This can therefore preclude the usage of BBD based RC schemes for very high-bandwidth positioning stages, and for very low-frequency reference signals. The BBD implementation is an exceptionally cheap solution that sometimes can be used to replace more expensive DSP solutions.

\subsubsection{Bucket Brigade Devices (BBDs)}

The BBD is used to realize the necessary time-delay. In a BBD the input signal is time-sampled and passed into a series of capacitors and transistor switches. The charge in each capacitor stage is passed into the subsequent stage at a rate determined by an external clock signal [37, 38, 39]. This delays the input signal. The BBD is a hybrid device that uses sampling, but not quantization. Low-noise BBDs from the MN3000-series from Panasonic can achieve a signal-to-noise ratio, or dynamic range, from 80 to $90 \mathrm{~dB}$. Using terminology from the analysis of ADCs, this equates to 13 or more effective number of bits (ENOBs). This is comparable to the actual performance when using 16bit ADCs [45], and also corresponds to the performance of the dSPACE DS1103 system. The dynamic range for a BBD can in some cases be improved by using companding (compressing and expanding a signal before and after some operation) [39], but this has not been applied in the presented implementation.

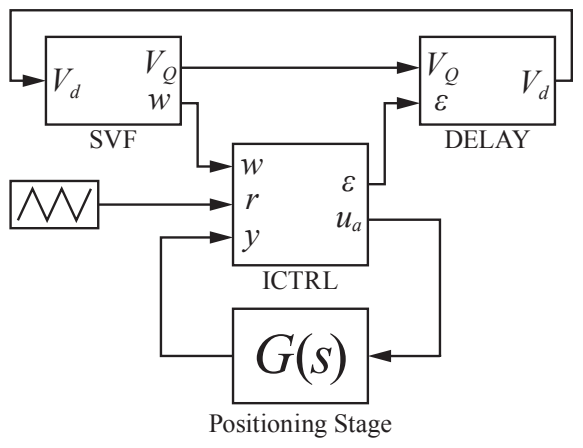

Figure 14: Interconnection of the different components of the implemented circuits, cf. Figs. 16a, 16b, and 16c.

Here the dynamic range has been maximized by linearly scaling the signals going in and out of the BBD. An alternative to BBDs is charge coupled device (CCD) delay lines [38, 46]. However, BBDs for audio applications are still in production and available for purchase at the time of writing.

\subsubsection{Overview}

The analog implementation of the control scheme consists of three parts: the time-delay; the integral control law and antialiasing and reconstruction filters; and the $Q(s)$ and $R(s)$ filters. The interconnection between these parts is shown in Fig. 14, and the actual implementation is shown in Fig. 15. In addition to the BBD, the necessary filters were implemented using standard operational amplifier circuits (the OPA227 was used in all circuits). An Agilent 33220A function generator was used to generate a reference, and a Tektronix TDS 3014C oscilloscope was used to record the results.

\subsubsection{Realization of State-Variable Filter}

The topology for a state-variable filter can be which implements the general stable, proper transfer-function

$$
\frac{Y(s)}{U(s)}=\frac{b_{0} s^{n}+b_{1} s^{n-1}+\ldots+b_{n-1} s+b_{n}}{s^{n}+a_{1} s^{n-1}+\ldots+a_{n-1} s+a_{n}},
$$

can be found following the development in [47]. Fig. 16a displays the fifth-order filter required to implement $Q(s)$ and $R(s)$. As $R(s)=W_{T}^{-1}(s) Q(s)$, and since $W_{T}(s)$ and $Q(s)$ are allpole filters, $Q(s)$ and $R(s)$ can therefore be realized using a single filter with two outputs.

The network analysis of the topology in the time-domain closely resembles a system on controllable canonical form, and

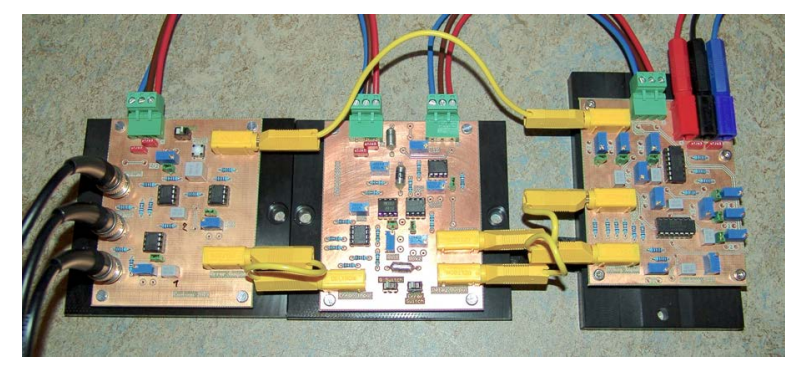

Figure 15: Implemented RC scheme. 


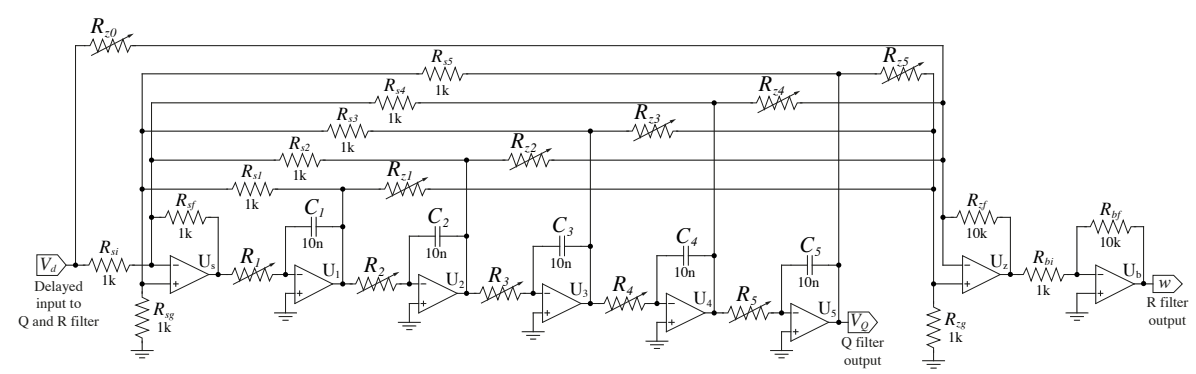

(a) State-variable filter with fives states, generating five poles and zeros.

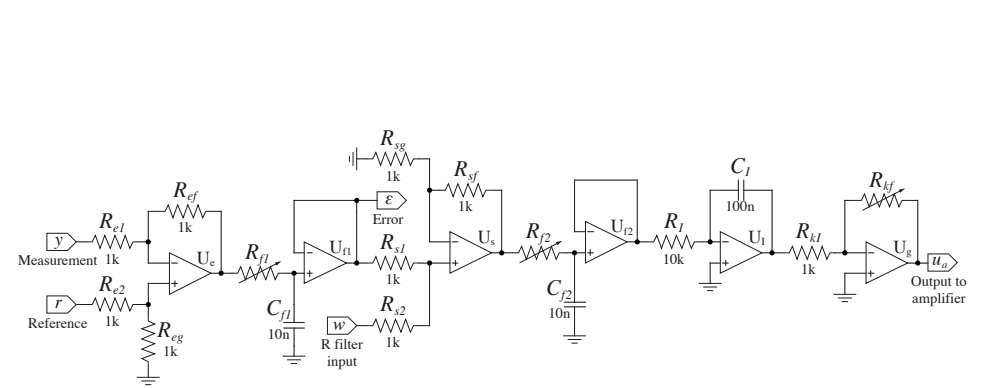

(b) Integral control law, anti-aliasing and reconstruction filter, and summation stage.

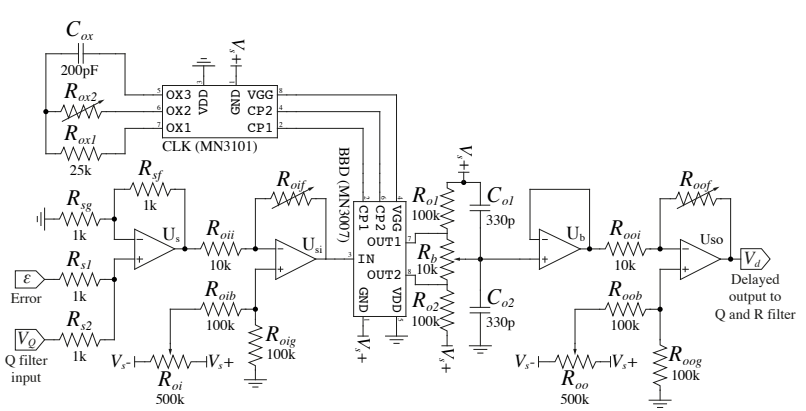

(c) Time-delay with offset and scaling.

Figure 16: Circuits for the realizations of the different parts in Fig. 14.

thus it is straight-forward to find the mapping from the coefficients $\left\{a_{i}\right\}$ and $\left\{b_{0}, b_{i}\right\}$, to the resistances $\left\{R_{i}\right\},\left\{R_{z_{0}}, R_{z_{i}}\right\}$, $i \in\{1,2,3,4,5\}$. Denoting the time-constant for each integrator in the filter

$$
\tau_{i}=R_{i} C_{i}
$$

the resistances $\left\{R_{i}\right\}$ determining the poles are found using:

$$
a_{1}=\frac{1}{\tau_{1}}, a_{2}=\frac{a_{1}}{\tau_{2}}, a_{3}=\frac{a_{2}}{\tau_{3}}, a_{4}=\frac{a_{3}}{\tau_{4}}, a_{5}=\frac{a_{4}}{\tau_{5}}
$$

The zeros of the filter are determined by the resistances of $\left\{R_{z 0}, R_{z i}\right\}$. Defining

$$
\begin{gathered}
G_{p}=1 / R_{z 1}+1 / R_{z 3}+1 / R_{z 5}+1 / R_{g 1}, \\
G_{n}=1 / R_{z 2}+1 / R_{z 4}+1 / R_{z 0}, \\
K_{p}=\left(1+R_{f 1} G_{n}\right) / G_{p}, \text { and } K_{n}=-R_{f 1},
\end{gathered}
$$

the required resistances can be found solving:

$$
\left[\begin{array}{c}
K_{p} / R_{z 1}+\left(R_{b f} / R_{b i}\right) \tau_{1}\left(b_{5}-a_{5} b_{0}\right) \\
K_{n} / R_{z 2}+\left(R_{b f} / R_{b i}\right) \tau_{1} \tau_{2}\left(b_{4}-a_{4} b_{0}\right) \\
K_{p} / R_{z 3}+\left(R_{b f} / R_{b i}\right) \tau_{1} \tau_{2} \tau_{3}\left(b_{3}-a_{3} b_{0}\right) \\
K_{n} / R_{z 4}+\left(R_{b f} / R_{b i}\right) \tau_{1} \tau_{2} \tau_{3} \tau_{4}\left(b_{2}-a_{2} b_{0}\right) \\
K_{p} / R_{z 5}+\left(R_{b f} / R_{b i}\right) \tau_{1} \tau_{2} \tau_{3} \tau_{4} \tau_{5}\left(b_{1}-a_{1} b_{0}\right) \\
K_{p} / R_{z 0}+\left(R_{b f} / R_{b i}\right) b_{0}
\end{array}\right]=\mathbf{0}
$$

In the general case, depending on the exact values for the poles and zeros, it might be necessary to modify the output summation stage generating the signal $w$, in order to find a realizable filter.

\subsubsection{Realization of Modified Integral Control Law}

The modified I control law is implemented as shown in Fig. 16b. The anti-aliasing and reconstruction filters are first- order low-pass, and the cut-off frequency $\omega_{c}$ is determined by adjusting $R_{f 1}$ and $R_{f 2}$ to satisfy

$$
\omega_{c}=1 / R_{f 1} C_{f 1}=1 / R_{f 2} C_{f 2} .
$$

The integral control law gain is determined, assuming $R_{e 1}=$ $R_{e 2}=R_{e f}=R_{e g}$ and $R_{s 1}=R_{s 2}$ and $R_{s f}=R_{s g}$, by adjusting $R_{k f}$ to satisfy

$$
k_{i}=R_{k f} / R_{k I} R_{I} C_{I}
$$

\subsubsection{Realization of Time-Delay}

The circuit implementing the time-delay is shown in Fig. 16c. The time-delay $\tau_{p}$ in the BBD is determined as

$$
\tau_{p}=N / 2 f_{c p}
$$

where $N$ is the number of stages in the BBD and $f_{c p}$ is the frequency of the external clock signal [38]. For the BBD used in the implementation, Panasonic MN3007, $N=1024$. As the BBD is a unipolar device with non-unity gain, the signal must be shifted and scaled before and after passing through the BBD. The shifting (bias) and scaling is adjusted using $R_{o i f}$, $R_{o i}, R_{o o f}$, and $R_{o o}$. The output of the BBD has a first-order low-pass filter with a cut-off frequency of approximately $\left(2 \pi \cdot 5 \cdot 10^{3} \cdot 330 \cdot 10^{-12}\right)^{-1}=96.5 \mathrm{kHz}$ to remove some of the clock signal noise. The frequency $f_{c p}$ can be adjusted monitoring either the CP1 or CP2 output of the MN3101 clock generator on an oscilloscope, while adjusting the resistance $R_{o x 2}$.

\subsection{Results and Discussion}

One result from the experiments when using the digital implementation is presented in Fig. 17. Here a $200 \mathrm{~Hz}$ modified 

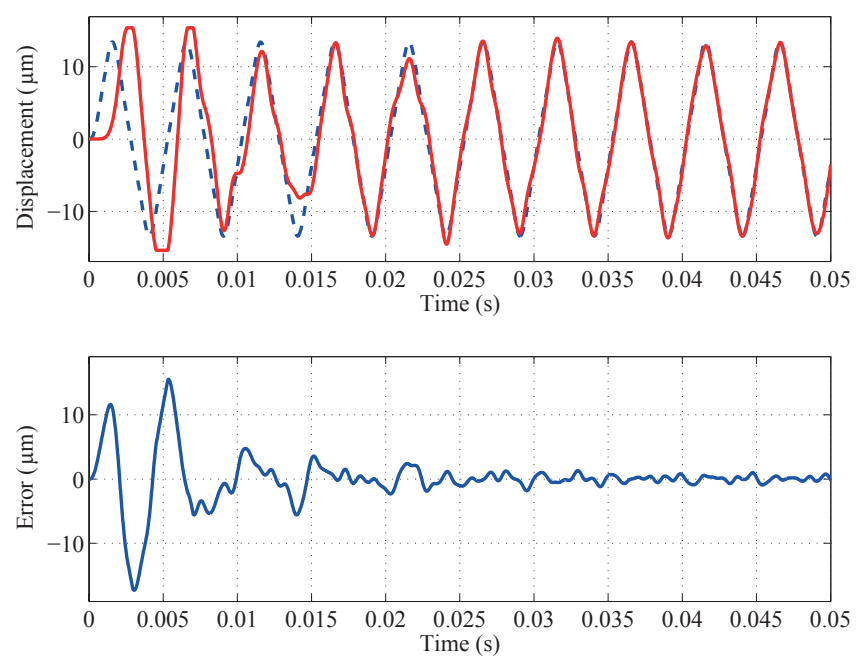

(a) Initial transient measured displacement $y_{m}$ and measured error $\varepsilon$.

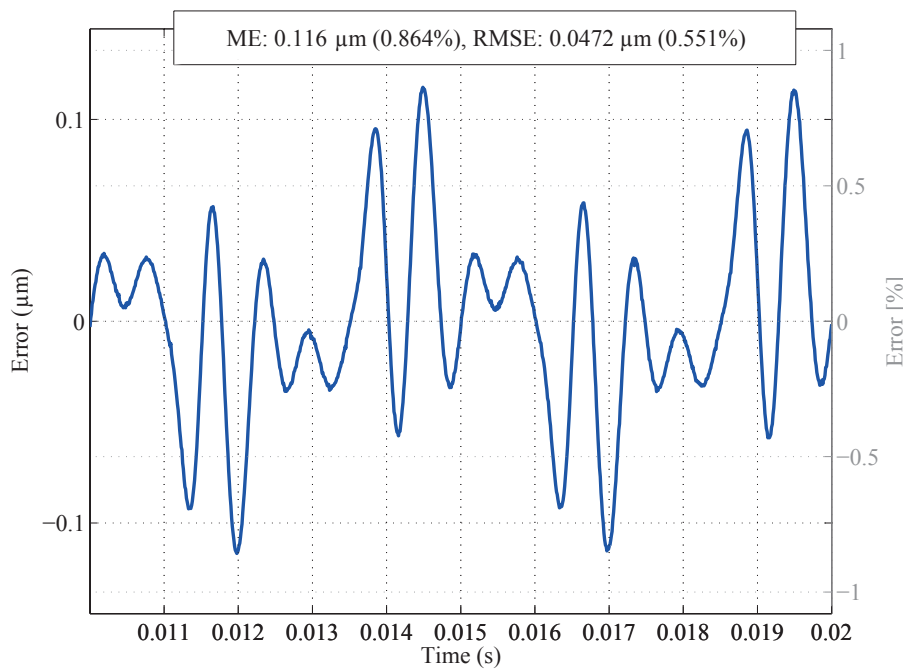

(c) Steady-state measured error $\varepsilon$.

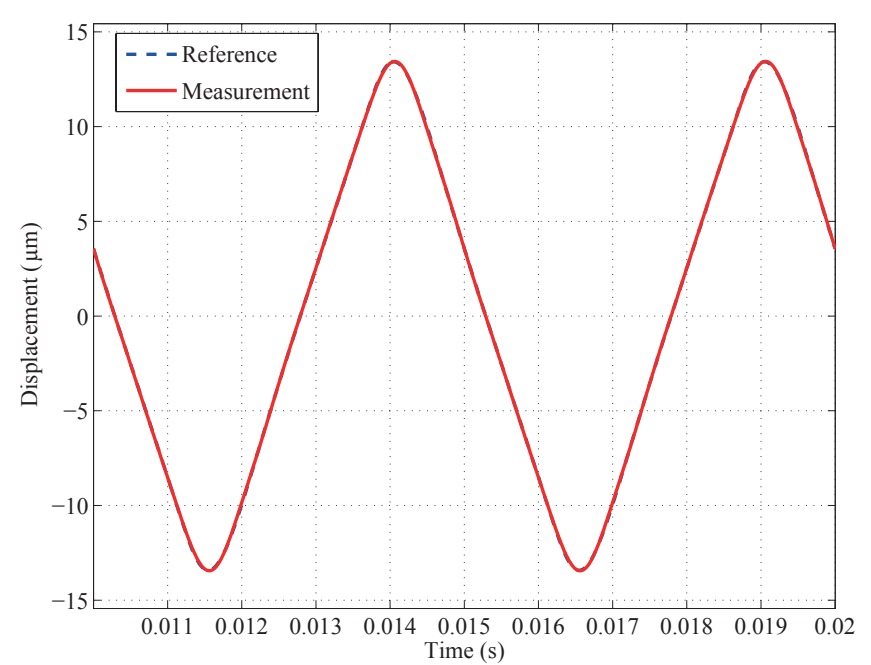

(b) Steady-state measured displacement $y_{m}$.

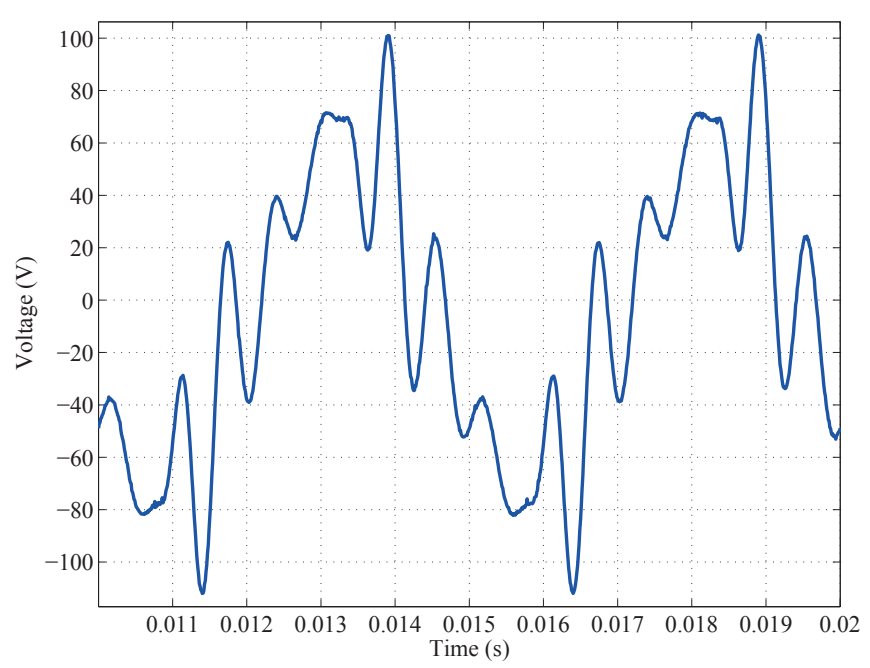

(d) Steady-state input voltage $u$.

Figure 17: Modified triangle-wave reference at $200 \mathrm{~Hz}$ with $14.25 \mu \mathrm{m}$ amplitude (27 $\mu \mathrm{m}$ linear range).

triangle-wave is used. Fig. 17a shows the initial transient response of the measured displacement and error, Fig. 17b the steady-state measured displacement, and Fig. 17c the steadystate measured error. In Fig. 17d the steady-state control input $u$ is shown. A summary of all the experiments performed using the digital implementation is presented in Tab. 5.

The results of the experiments in Tab. 5 are rated using both the maximum error (ME) and the root-mean-square error (RMSE), both in absolute terms with respect to the measured displacement, and relative to the maximum value of the reference for ME, and the RMS value of the reference for RMSE.

Tracking results when using the analog implementation is presented in Fig. 18. The main problem with this implementation is that the state-variable filter topology has high sensitivity to the component values in the summation stage generating the zeros. To reduce the sensitivity, the filters could be implemented using biquadratic [48] stages. This would be at the cost of having two separate filters instead of one single filter with one input and two outputs, since it would no longer be possible to sum all the states to generate the zeros and have a separate path for the low-pass filter response. The digital implementation is much more precise in terms of achieving specified filter response. An equivalent of the analog implementation was therefore implemented on the dSPACE system to serve as a reference to be matched. Tracking results using the digital equivalent is shown in Fig. 18a, and tracking results when using the actual analog implementation is shown Fig. 18b. As can be seen, it closely matches the digital equivalent in terms of tracking performance. As a regular triangle-wave was used, the maximum error is larger than if the modified triangle-wave had been used.

With regards to the $\mathrm{RC}$ performance, as can be seen from Tab. 5, the closed-loop error using references with low fundamental frequency yields errors close to the closed-loop measurement noise, i.e. in practical terms, perfect tracking is achieved. The performance depend mostly on the bandwidth of the filter $Q(s)$. A larger $k_{T}$ results in amplification of measurement 
Table 5: Norms of the measured error $\varepsilon$ in steady-state (digital impl.), for various configurations and references. $f_{p}$ and $2 \lambda$ denotes the fundamental frequency and linear range of the reference signal, respectively.

\begin{tabular}{cccccccc}
\hline \hline$f_{p}$ & $2 \lambda$ & $k_{T}$ & $\begin{array}{c}f_{Q} \\
(\mathrm{kHz})\end{array}$ & $\begin{array}{c}\text { ME } \\
\text { abs. } \\
(\mu \mathrm{m})\end{array}$ & $\begin{array}{c}\text { ME } \\
\text { rel. } \\
(\%)\end{array}$ & $\begin{array}{c}\text { RMSE } \\
\text { abs. } \\
(\mu \mathrm{m})\end{array}$ & $\begin{array}{c}\text { RMSE } \\
\text { rel. } \\
(\%)\end{array}$ \\
\hline
\end{tabular}

When using optimally tuned PI control law (without RC scheme):

$\begin{array}{cccccccc}25 & 27.0 & - & - & 1.7 & 12 & 1.0 & 12 \\ 200 & 27.0 & - & - & 16 & 120 & 9.8 & 110\end{array}$

When using optimally tuned PI control law and RC scheme:

\begin{tabular}{ccllllll}
25 & 13.5 & 0.450 & 2 & 0.0034 & 0.048 & 0.00067 & 0.016 \\
25 & 27.0 & 0.350 & 2 & 0.0048 & 0.033 & 0.0011 & 0.013 \\
25 & 27.0 & 0.450 & 2 & 0.0072 & 0.050 & 0.0017 & 0.020 \\
50 & 27.0 & 0.450 & 2 & 0.011 & 0.077 & 0.0028 & 0.032 \\
100 & 27.0 & 0.450 & 2 & 0.031 & 0.22 & 0.010 & 0.12 \\
200 & 13.5 & 0.450 & 2 & 0.61 & 0.91 & 0.025 & 0.59 \\
200 & 27.0 & 0.450 & 2 & 0.12 & 0.86 & 0.047 & 0.55 \\
400 & 13.5 & 0.450 & 2 & 0.12 & 1.9 & 0.064 & 1.6 \\
\hline
\end{tabular}

noise. Some of the error at low fundamental frequencies is due to the quantization noise, as the DAC noise floor is reached within the bandwidth of the RC. The gain $k_{T}$ also determines the time-constant for the transient response envelope, thus larger $k_{T}$ results in faster convergence to steady-state.

In Fig. 17a the transient response and convergence to steadystate is shown. There is some saturation in the measurement, leading to longer convergence time. From Fig. 17c it can be seen that the the maximum error occurs at the extrema of the reference, thus, increasing the linear proportion of the reference will also increase the maximum error. The voltage range of the signal in Fig. 17d should be noted. Here the full range of the amplifier, $\pm 110 \mathrm{~V}$, is used and provides a hard limit for the spectral content and the range of the reference, as well as the bandwidth of the control law. Increasing either one of these properties would have resulted in saturation in the amplifier.

The achieved performance depend mostly on the bandwidth of the filter $Q(s)$, as discussed in Sec. 4.2.2. To increase performance, a zero-phase filter can be used. For a digital implementation, this is possible both for ILC [25] and RC [11]. It is not possible for an analog implementation. As the bandwidth of $Q(s)$ in this work is limited by higher order non-modeled vibration modes, it is likely not possible to improve the performance without using model matching and subsequently increase the order of the control law filters. However, experimental results for a similar experimental system using discrete-time RC can be found in [11]. Comparing the results, the presented continuous-time scheme will perform on par with the common discretetime RC implementation that uses high-order zero-phase tracking error control (ZPETC) model-inversion and signal model bandwidth limitation using zero-phase filtering [42, 20]. The discrete-time implementation in [11] achieved an ME of $0.83 \%$ and an RMSE of $0.10 \%$ for a $40-\mathrm{Hz}$ triangle wave reference signal with 5- $\mu \mathrm{m}$ amplitude when there is a dominant vibration mode at $520 \mathrm{~Hz}$, compared to an ME of $0.634 \%$ and an RMSE of $0.307 \%$ for a $50-\mathrm{Hz}$ triangle wave reference signal with $15-\mu \mathrm{m}$

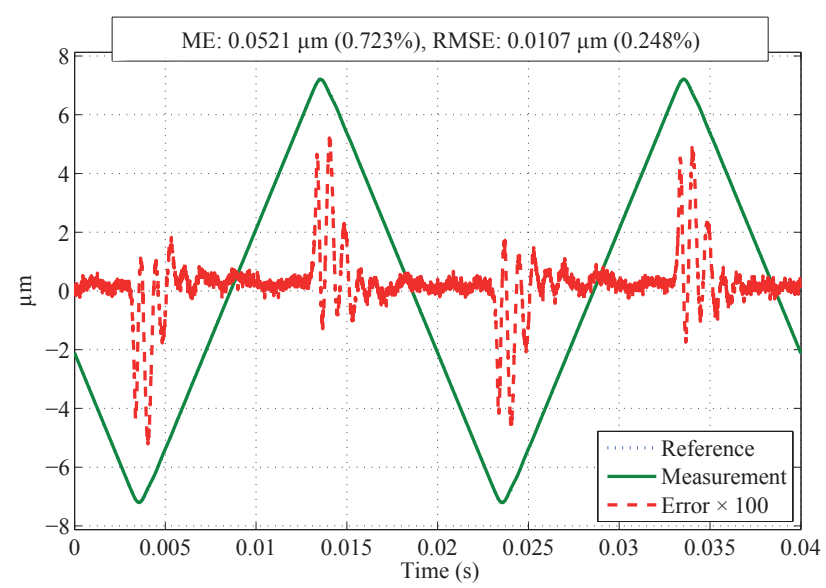

(a) Equivalent of analog implementation on dSPACE system.

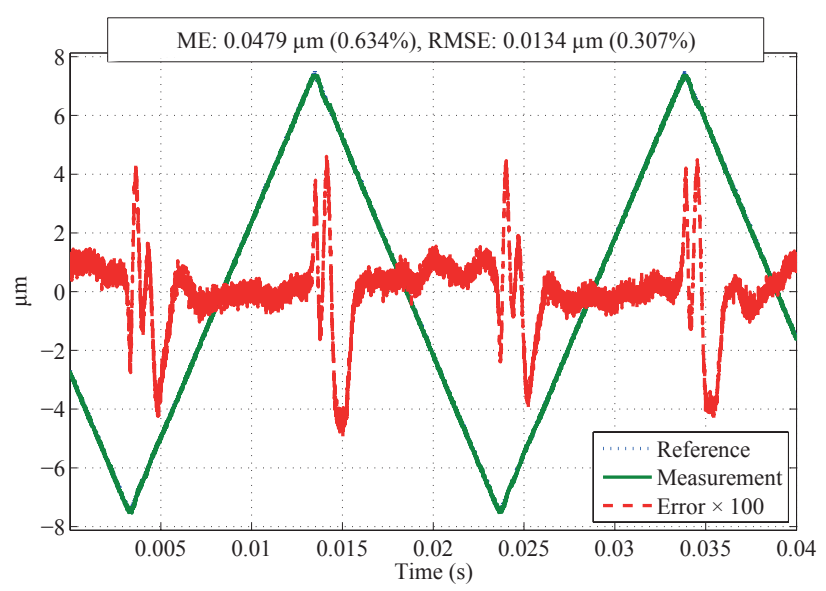

(b) Analog implementation.

Figure 18: Performance of the analog RC implementation. Regular trianglewave reference at $50 \mathrm{~Hz}$ with $15 \mu \mathrm{m}$ range.

amplitude when there is a dominant vibration mode at $704 \mathrm{~Hz}$ for the analog implementation in this work.

\section{Conclusions}

It has been demonstrated through experiments that, by using a control scheme combining a damping and tracking control law and continuous-time plug-in repetitive control, it is possible to achieve a maximum error of less than $1 \%$ relative to the reference, at reference signal frequencies exceeding $25 \%$ of the first resonance frequency of a nanopositioning stage. Analog and digital implementations were presented. The analog implementation was feasible due to the low order of the scheme, and provides an inexpensive realization with high performance. The low order also enables fast and efficient digital implementation. The control scheme is straight-forward to tune using optimization techniques, and is applicable for tracking control on flexible smart structures in general. The proposed scheme is implementable on existing hardware configurations for nanopositioning, as it requires very small, or no, modifications to a standard configuration. 


\section{Acknowledgments}

This work was supported by the Norwegian University of Science and Technology, and in part by the National Science Foundation grant CMMI No. 0910570. Authors thank Brian J. Kenton for his help with developing the flexure-based nanopositioning stage used in the experiments.

[1] S. M. Salapaka, M. V. Salapaka, Scanning Probe Microscopy, IEEE Control Syst. Mag. 28 (2) (2008) 65-83.

[2] G. M. Clayton, S. Tien, K. K. Leang, Q. Zou, S. Devasia, A Review of Feedforward Control Approaches in Nanopositioning for High-Speed SPM, J. Dyn. Syst.-T. ASME 131 (6) (2009) 061101 (19 pages).

[3] A. Biswas, I. S. Bayer, A. S. Biris, T. Wang, E. Dervishi, F. Faupel, Advances in top-down and bottom-up surface nanofabrication: Techniques, applications \& future prospects, Adv. Colloid Interface Sci. 170 (1-2) (2012) 2-27.

[4] B. A. Francis, W. M. Wonham, Internal Model Principle of ControlTheory, Automatica 12 (5) (1976) 457-465.

[5] T. Inoue, M. Nakano, S. Iwai, High accuracy control of a proton synchrotron magnet power supply, in: Proc. 8th World Congr. IFAC, Vol. 20, 1981 , pp. $216-221$.

[6] S. Hara, Y. Yamamoto, T. Omata, M. Nakano, Repetitive Control System: A New Type Servo System for Periodic Exogenous Signals, IEEE Trans. Autom. Control 33 (7) (1988) 659-668.

[7] Y. Yamamoto, S. Hara, Internal and External Stability and Robust Stability Condition for a Class of Infinite-Dimensional Systems, Automatica 28 (1) (1992) 81-93.

[8] U. Aridogan, Y. Shan, K. K. Leang, Design and Analysis of DiscreteTime Repetitive Control for Scanning Probe Microscopes, J. Dyn. Syst.-T. ASME 131 (6) (2009) 061103 (12 pages).

[9] R. J. E. Merry, D. J. Kessels, W. P. M. H. Heemels, M. J. G. van de Molengraft, M. Steinbuch, Delay-varying repetitive control with application to a walking piezo actuator, Automatica 47 (8) (2011) 1737-1743.

[10] Y. Shan, K. K. Leang, Design and Control for High-Speed Nanopositioning: Serial-Kinematic Nanopositioners and Repetitive Control for Nanofabrication, IEEE Control Syst. Mag. 33 (6) (2013) 86-105.

[11] Y. R. Teo, A. A. Eielsen, J. T. Gravdahl, A. J. Fleming, Discrete-time repetitive control with model-less FIR filter inversion for high performance nanopositioning, in: P. IEEE/ASME Int. Conf. Adv. Intell. Mechatron., 2014, pp. 1664-1669.

[12] Y. K. Yong, S. O. R. Moheimani, B. J. Kenton, K. K. Leang, Invited Review Article: High-speed flexure-guided nanopositioning: Mechanical design and control issues, Rev. Sci. Instrum. 83 (12) (2012) 121101 (22 pages).

[13] A. A. Eielsen, J. T. Gravdahl, K. Y. Pettersen, Adaptive feed-forward hysteresis compensation for piezoelectric actuators, Rev. Sci. Instrum. 83 (8) (2012) 085001 (8 pages).

[14] M. Steinbuch, S. Weiland, T. Singh, Design of Noise and Period-Time Robust High-Order Repetitive Control, with Application to Optical Storage, Automatica 43 (12) (2007) 2086-2095.

[15] G. Pipeleers, B. Demeulenaere, J. De Schutter, J. Swevers, Robust HighOrder Repetitive Control, in: P. Amer. Contr. Conf., 2008, pp. 1080-1085.

[16] R. J. E. Merry, M. J. C. Ronde, R. van de Molengraft, K. R. Koops, M. Steinbuch, Directional Repetitive Control of a Metrological AFM, IEEE Trans. Control Syst. Technol. 19 (6) (2011) 1622-1629.

[17] T. E. Peery, H. Özbay, H $\infty$ Optimal Repetitive Controller Design for Stable Plants, J. Dyn. Syst.-T. ASME 119 (3) (1997) 541-547.

[18] J. Li, T.-C. Tsao, Robust Performance Repetitive Control Systems, J. Dyn. Syst.-T. ASME 123 (3) (2001) 330-337.

[19] K. Yamada, T. Arakawa, H. Hoshi, T. Okuyama, Two-Step Design Method for Robust Repetitive Control Systems, JSME Int J., Ser. C 46 (3) (2003) 1068-1074

[20] A. W. Osburn, M. A. Franchek, Designing Robust Repetitive Controllers, J. Dyn. Syst.-T. ASME 126 (4) (2004) 865-872.

[21] S. Skogestad, I. Postlethwaite, Multivariable Feedback Control: Analysis and Design, Wiley-Interscience, 2005.

[22] K. L. Moore, M. Dahleh, S. P. Bhattacharyya, Iterative Learning Control: A Survey and New Results, J. Robotic Syst. 9 (5) (1992) 563-594.

[23] K. K. Leang, S. Devasia, Design of Hysteresis-Compensating Iterative
Learning Control for Piezo-Positioners: Application to Atomic Force Microscopes, Mechatronics 16 (2006) 141-158.

[24] Y. Wu, Q. Zou, Iterative Control Approach to Compensate for Both the Hysteresis and the Dynamics Effects of Piezo Actuators, IEEE Trans. Control Syst. Technol. 15 (5) (2007) 936-944.

[25] D. A. Bristow, J. Dong, A. G. Alleyne, P. Ferreira, S. Salapaka, High bandwidth control of precision motion instrumentation, Rev. Sci. Instrum. 79 (10) (2008) 103704 (14 pages).

[26] A. A. Eielsen, J. T. Gravdahl, K. K. Leang, Robust Damping PI Repetitive Control for Nanopositioning, in: P. Amer. Contr. Conf., 2012, pp. 38033810 .

[27] A. A. Eielsen, J. T. Gravdahl, K. K. Leang, Analog Robust Repetitive Control for Nanopositioning Using Bucket Brigade Devices, in: P. 19th IFAC World Congr., 2014, pp. 1126-1133.

[28] Y. Shan, K. K. Leang, Accounting for Hysteresis in Repetitive Control Design: Nanopositioning Example, Automatica 48 (8) (2012) 1751-1758.

[29] A. A. Eielsen, M. Vagia, J. T. Gravdahl, K. Y. Pettersen, Damping and Tracking Control Schemes for Nanopositioning, IEEE/ASME Trans. Mechatronics 19 (2) (2014) 432-444.

[30] A. J. Fleming, S. O. R. Moheimani, Sensorless Vibration Suppression and Scan Compensation for Piezoelectric Tube Nanopositioners, IEEE Trans. Control Syst. Technol. 14 (1) (2006) 33-44.

[31] S. S. Aphale, B. Bhikkaji, S. O. R. Moheimani, Minimizing Scanning Errors in Piezoelectric Stack-Actuated Nanopositioning Platforms, IEEE Trans. Nanotechnol. 7 (1) (2008) 79-90.

[32] A. Sebastian, A. Pantazi, S. O. R. Moheimani, H. Pozidis, A Self Servo Writing Scheme for a MEMS Storage Device with Sub-nanometer Precision, in: P. 17th IFAC World Congr., 2008, pp. 9242-9247.

[33] B. Bhikkaji, S. O. R. Moheimani, Integral Resonant Control of a Piezoelectric Tube Actuator for Fast Nanoscale Positioning, IEEE/ASME Trans. Mechatronics 13 (5) (2008) 530-537.

[34] C. Lee, S. M. Salapaka, Robust broadband nanopositioning: fundamental trade-offs, analysis, and design in a two-degree-of-freedom control framework, Nanotechnology 20 (2009) 035501 (16 pages).

[35] A. J. Fleming, Nanopositioning System With Force Feedback for HighPerformance Tracking and Vibration Control, IEEE/ASME Trans. Mechatronics 15 (3) (2010) 433-447.

[36] S. Butterworth, On the Theory of Filter Amplifiers, Experimental Wireless \& The Wireless Engineer 7 (1930) 536-541.

[37] F. L. J. Sangster, K. Teer, Bucket-Brigade Electronics- New Possibilities for Delay, Time-Axis Conversion, and Scanning, IEEE J. Solid-State Circuits 4 (3) (1969) 131-136.

[38] G. Weckler, Bucket Brigade Devices Circa 1977, in: Charge Transfer Device Signal Processing - A Short Course Organized by M. H. White, The American University, 1977.

[39] C. Raffel, J. Smith, Practical Modeling of Bucket-Brigade Device Circuits, in: P. 13th Int. Conf. on Digital Audio Effects, 2010.

[40] J. Leyva-Ramos, G. Escobar, P. R. Martinez, P. Mattavelli, Analog Circuits to Implement Repetitive Controllers for Tracking and Disturbance Rejection of Periodic Signals, IEEE Trans. Circuits Syst. II 52 (8) (2005) 466-470.

[41] G. Escobar, P. R. Martinez, J. Leyva-Ramos, Analog Circuits to Implement Repetitive Controllers With Feedforward for Harmonic Compensation, IEEE Trans. Ind. Electron 54 (1) (2007) 567-573.

[42] M. Tomizuka, T.-C. Tsao, K.-K. Chew, Analysis and Synthesis of Discrete-Time Repetitive Controllers, J. Dyn. Syst.-T. ASME 111 (3) (1989) 353-358.

[43] B. J. Kenton, K. K. Leang, Design and Control of a Three-Axis SerialKinematic High-Bandwidth Nanopositioner, IEEE/ASME Trans. Mechatronics 17 (2) (2012) 356-369.

[44] T. I. Laakso, V. Välimäki, M. Karjalainen, U. K. Laine, Splitting the Unit Delay, IEEE Signal Process. Mag. 13 (1) (1996) 30-60.

[45] R. H. Walden, Analog-to-Digital Converter Survey and Analysis, IEEE J. Sel. Areas Commun. 17 (4) (1999) 539-550.

[46] J. A. Catrysse, G. Declerck, C. C. De Meyer, CCD Delay Lines in Audio, J. Audio Eng. Soc. 28 (11) (1980) 800-808.

[47] W. J. Kerwin, L. P. Huelsman, R. W. Newcomb, State-Variable Synthesis for Insensitive Integrated Circuit Transfer Functions, IEEE J. Solid-State Circuits 2 (3) (1967) 87-92.

[48] J. Tow, Active RC filters-A state-space realization, Proc. IEEE 56 (6) (1968) 1137-1139. 